\title{
SPACES OF IDEALS OF DISTRIBUTIVE LATTICES II. MINIMAL PRIME IDEALS
}

\author{
T. P. SPEED
}

(Received 2 April 1970; revised 11 November 1970)

Communicated by B. Mond

\section{Introduction}

This paper, the second of a sequence beginning with [14], deals with the relationship between a distributive lattice $\mathbf{L}=(L ; \vee, \wedge, 0)$ with zero, and certain spaces of minimal prime ideals of $\mathbf{L}$. Similar studies of minimal prime ideals in commutative semigroups [8] and in commutative rings [6] inspired this work, and many of our results are similar to ones in these two articles. However the nature of our situation enables many of these results to be pushed deeper and thus to arrive at a more satisfactory state; indeed with the insight obtained from the simpler lattice situation, one can return to some topics considered in [6], [8] and give complete accounts. We do not do this in the present paper, but leave the details to the reader, see e.g. [15]. Also a study of minimal prime ideals illuminates some topics in the theory of distributive lattices, particularly Stone lattices.

After some preliminaries we present the basic properties of the spaces Minp L, including a characterisation. Next some homeomorphism and isomorphism theorems are given, followed by a brief discussion of functorial aspects. Our main material comprises detailed discussions of the various forms of compactness and connectedness which the spaces may exhibit, and we close the paper with some examples which illustrate the theory and show the necessity of most of our hypotheses.

\section{Preliminaries}

All lattices in this paper are distributive and possess a zero.

1.1. For the basic facts concerning minimal prime ideals belonging to an ideal $J$ of $\mathbf{L}$ we refer to [5]. The set of all minimal prime ideals belonging to the ideal .$J$ of $\mathbf{L}$ is denoted $\mathscr{M}_{\boldsymbol{L}}^{J}$; in the case $J=(0)$ we call the ideals minimal prime, and 
write the set of all minimal prime ideals of $\mathbf{L}$ as $\mathscr{M}_{\mathbf{L}}$. All hulls $h(\cdot)$ and kernels $k(\cdot)$ (see [5]) refer to $\mathscr{M}_{L}$.

1.2. The principal ideal of $\mathbf{L}$ generated by $a \in L$ is written $(a)_{L}$. For two subsets $A, B$ of $L$ we put $(A: B)_{L}^{*}=\{t \in L: t \wedge a \in B$ for all $a \in A\}$. If $B=\{0\}$ we write $A_{L}^{*}$ instead of $(A:\{0\})_{L}^{*}$ and when $A=\{a\}$ and $B=\{0\}$ we write $(a)_{L}^{*}$ instead of $(\{a\},\{0\})_{\mathbf{L}}^{*}$. Also we put $A_{L}^{* *}=\left(A_{L}^{*}\right)_{L}^{*}$. The dual ideal of dense elements of $\mathbf{L}$ is $D_{\boldsymbol{L}}=\left\{d \in L:(d)_{L}^{*}=(0)\right\}$; we also have occasion to use $D_{\mathbf{L}}^{J}$ $=\left\{d \in L:(d: J)_{L}^{*}=J\right\}$, where $J$ is an ideal of $\mathbf{L}$.

1.3. Basic to what follows is the characterisation of minimal prime ideals: a prime ideal $M \supset J$ containing the ideal $J$ of $\mathbf{L}$ is a minimal prime ideal belonging to $J$ iff for any $x \in M$ there is $y \notin M$ such that $x \wedge y \in J$. In the case $J=(0)$ we obtain: a prime ideal $M$ is minimal iff for any $x \in M,(x)^{*} \mid M$ is non-empty. If $M$ is a minimal prime of $\mathbf{L}$ then : $x \in M$ iff $(x)^{* *} \subset M ; x \notin M$ iff $(x)^{*} \subset M$. We also need the result: $k\left(\left\{M \in \mathscr{M}_{\mathbf{L}}: M \nsubseteq A\right\}\right)=A_{L}^{*}$ for any $A \subset L$. All of these results can be found in [8]. Finally it is shown in [14] that there is an order isomorphism between the prime ideals not containing an ideal $J$ of $L$ and the prime ideals of $J$ as a lattice. The correspondence is $P \rightarrow P \cap J$ with inverse $P_{1}$ $\rightarrow\left(J: P_{1}\right)_{L}^{*}$.

1.4. For basic facts concerning distributive lattices we refer to $[1]$; there the least congruence $\Theta^{J}$ having a given ideal $J$ as congruence class is defined. We will write $L / J$ instead of $L / \Theta^{J}$. The empty set is denoted $\phi$, the set difference of $A$ and $B$ by $A \backslash B$, and the cardinality of a set $A$ by $|A|$.

1.5. Subscripts in the expressions such as $\mathscr{M}_{L},(x)_{L}$ are only included when the $\mathbf{L}$ in question needs to be emphasised; otherwise we write $\mathscr{M},(x)$.

\section{Basic properties of Minp L}

Equip $\mathscr{M}=\mathscr{M}_{\boldsymbol{L}}$ with the topology induced by the closure operator $\mathscr{A} \rightarrow \mathscr{A}^{-}=h(k(\mathscr{A}))$. The resulting space is denoted Minp L and called the minimal spectrum of $\mathbf{L}$. (Other writers have called this space the space of minimal prime ideals, but since we propose to discuss the dual topology, it seems preferable to avoid this usage.) The following have their easy proofs omitted.

Proposition 2.1. For any ideal $J$ of $\mathbf{L}$ the set $\mathscr{M}(J)=\{M \in \mathscr{M}: M \ngtr J\}$ is open in Minp L. Further, the family $\mu_{\mathrm{L}}=\{\mathscr{M}(x): x \in L\}$ where $\mathscr{M}(x)=\mathscr{M}((x))$, forms $a$ (join) basis for the open sets of Minp $\mathbf{L}$.

Corollary 2.2. For any ideal $J$ of $\mathbf{L}$ the set $h(J)=\{M \in \mathscr{M}: M \supset J\}$ is closed in MinpL. Further, the family $\{h(x): x \in L\}$ forms a (meet) basis for the closed sets of Minp $\mathbf{L}$. 
Proposition 2.3. Minp $\mathbf{L}$ is a Hausdorff space.

Proof. For distinct minimal prime ideals $M$ and $N$ choose $x \in M \backslash N$; by 1.3 , there exists $y \in N \mid M$ such that $x \wedge y=0$. Thus $M \in \mathscr{M}(y)$ and $N \in \mathscr{M}(x)$ and

$$
\mathscr{M}(x) \cap \mathscr{M}(y)=\mathscr{M}(0)=\phi .
$$

The family $\mu_{L}$ in 2.1 forms a lattice which we call the dual lattice, and study more in $\S 3$.

An alternative topology can be defined on $\mathscr{M}$ which we call the dual spectral topology; it may be defined as that topology induced by the closure operator

$$
\mathscr{A} \rightarrow \mathscr{A}^{-}=\{M \in \mathscr{M}: M \subset \bigcup \mathscr{A}\}
$$

although we do not prove this fact. Instead we define it as the topology generated by the family $\{h(x): x \in L\}$; immediately dual results to $2.1,2.2$ can be written down. We denote this space by $\operatorname{Minp} L$ and remark that it is canonically homeomorphic with the space of maximal ideals of the dual lattice $\breve{L}$ equipped with the spectral topology.

By a quasi-compact topological space we mean (following [2], Part $1 \mathrm{pp}$. 83-84) a (not-necessarily-Hausdorff) space whose topology satisfies the usual covering axiom: every open cover possesses a finite subcover.

Proposition 2.4. The space Minp $\mathbf{L}$ has an open basis such that

(i) each basis set is open-closed, and

(ii) the topology generated by the complements of these basic open sets is quasi-compact.

Proof. The basis is of course $\mu_{L}=\{\mathscr{M}(x): x \in L\}$. For by $1.3 \mathscr{M}(x)=h\left((x)^{*}\right)$, and so each of the sets $\mathscr{M}(x)$ is open-closed by 2.1, 2.2. Also $\mathscr{M} \backslash \mathscr{M}(x)=h(x)$ and so the second assertion is just the statement that $M \operatorname{Minp} L$ is quasi-compact. Let $\mathscr{F}=\left\{\mathscr{C}_{i}: i \in I\right\}$ be a family of sets closed in Minp $\mathbf{L}$ and having the f.i.p. Then as each $\mathscr{C}_{i}$ is of the form $\bigcap\left\{\mathscr{M}(x): x \in A_{i}\right\}$ for $A_{i} \subset L$ it suffices to consider a family $\mathscr{F}^{\prime}=\{\mathscr{M}(x): x \in A\}$ with the f.i.p., requiring $\bigcap_{\mathscr{F}^{\prime}} \neq \phi$. But the method of [8] Lemma 7.2 gives an $M$ with $M \in \cap\{\mathscr{M}(x: x \in A\}$ and the proof is complete.

Proposition 2.5. The identity map 1: $\mathscr{M}_{\boldsymbol{L}} \rightarrow \mathscr{M}_{\mathbf{L}}$ defines a continuous bijection from Minp $\mathbf{L}$ onto Minp $\mathbf{L}$ i.e. the spectral topology is finer than the dual spectral topology.

Proof. The basis sets $h(x)$ of Minp $\mathbf{L}$ are open in $\operatorname{Minp} \mathbf{L}$ and so the result follows.

A Hausdorff space with an open basis consisting of open-closed sets is called zero-dimensional ([2], Part 2., p. 200): thus we have from 2.3, 2.4 (i). 
Corollary 2.6. Minp $\mathbf{L}$ is zero dimensional.

Motivated by the preceding results we define a Hausdorff topological space $(X, \mathscr{T})$ to be a $\zeta$-space if $\mathscr{T}$ possesses a basis $\mathscr{E}$ (which we call a $\zeta$-basis) satisfying (i) and (ii) of 2.4. Exactly as in 2.5 we note that every $\zeta$-space is zero-dimensional, hence the designation.

Our main result on $\zeta$-spaces is a converse to 2.4 which was obtained jointly with Mr. J. H. Rubinstein.

Proposition 2.7. Let $(X, \mathscr{T})$ be a $\zeta$-space with $\zeta$-basis $\mathscr{E}$. Then if $\mathscr{E}$ is the lattice generated by $\mathscr{E}^{\prime}$, Minp $\mathscr{E} \cong(X, \mathscr{T})$.

PRoOF. It is clear that we may without loss of generality assume $\mathscr{E}=\mathscr{E}$. Define a map $\tau: \mathscr{M}_{g} \rightarrow X$ by $M \tau=\bigcap\{a: a \notin M\}$; we will see that $\tau$ is well defined. Firstly, each $a \in \mathscr{E}$ is closed in the topology $\widetilde{\mathscr{T}}$ generated by $\{X \mid a: a \in \mathscr{E}\}$, and as the latter is quasi-compact (by assumption) and $\{a: a \notin M\}$ has the f.i.p., we deduce that $\cap\{a: a \notin M\} \neq \phi$. We next show that this intersection is a onepoint set. If $\xi \neq \xi^{\prime}$ are distinct points of the intersection, there is $b, b^{\prime}$ in $\mathscr{E}$ such that $b \cap b^{\prime}=\phi$ and $\xi \in b, \xi^{\prime} \in b^{\prime}$. Thus (by the prime property) one of $b, b^{\prime}$ is in $M$, say $b$; by 1.3 there is $c \notin M$ with $b \cap c=\phi$. Clearly $\xi \in c$, and this contradicts our assumption. Hence $\cap\{a: a \in M\}$ is a unique point and the map $\tau$ is welldefined.

We now observe that for any $\xi \in X, M(\xi)=\{a \in \mathscr{E}: \xi \notin a\}$ is a minimal prime. It is easy to see that $M(\xi)$ is prime; or the minimality take $a \in M(\xi)$, then $\xi \notin a$ and there is $b \subset X \backslash a$ with $\xi \in b$ and $b \in \mathscr{E}$. For this $b, b \notin M(\xi)$ and $a \cap b=\phi$ and so 1.3 applies.

It is now clear that

and

$$
M(\xi)=\{a \in \mathscr{E}: \bigcap\{b: b \notin M(\xi)\} \notin a\}
$$

proving that $\tau$ is bijective.

$$
\{\xi\}=\bigcap\{a: a \notin M(\xi)\}
$$

Finally the fact that $\tau$ and $\tau^{-1}$ are continuous is readily seen since the basic open sets $a \in \mathscr{E}$ and $\left\{M \in \mathscr{M}_{\mathscr{E}}: a \notin M\right\}$ correspond bijectively.

Thus the proof is complete.

Some further results on $\zeta$-spaces (including answers or counterexamples to some obvious conjectures) appear in [9]. We close this section with one more general result on $\zeta$-spaces. Recall ([2], Part 2, p. 192) that a topological space $X$ is called a Baire space if every countable intersection of dense open sets in $X$ is dense in $X$.

Proposition 2.8. Every $\zeta$-space is a Baire space.

Proof. Let $(X, \mathscr{T})$ be a $\zeta$-space with $\zeta$-basis $\mathscr{E} \subset \mathscr{T}$ assumed to be a lattice, and suppose $\left\{\mathscr{G}_{n}\right\}$ is a sequence of dense open sets in $(X, \mathscr{T})$. Then 
$\mathscr{G}_{n}=\bigcup\left\{a: a \in \mathscr{E}_{n}\right\}$ for $\mathscr{E}_{n} \subset \mathscr{E}$ and the denseness of $\mathscr{G}_{n}$ implies that for any $\phi \neq b \in \mathscr{E}$ there is a $a \in \mathscr{G}_{n}$ with $a \cap b \neq \phi$. We will now show $\bigcap_{n} \mathscr{G}_{n}$ dense. Take $x \in X$ and a neighbourhood $b$ of $x$, and we define recursively a sequence $\left\{a_{n}: n=0,1, \cdots\right\} \subset \mathscr{E}$. Put $a_{0}=b$ and suppose $a_{n-1}$ is defined such that $b \cap a_{1} \cap \cdots \cap a_{n-1} \neq \phi$; then take $a_{n} \in \mathscr{G}_{n}$ such that $b \cap a_{1} \cap \cdots \cap a_{n} \neq \phi$. The sequence $\left\{a_{n}: n=0,1, \cdots\right\}$ clearly has the f.i.p. and consists of sets closed in the topology generated by $\{X \mid a: a \in \mathscr{E}\}$ and thus

as required.

$$
\phi \neq \bigcap_{n=0}^{\infty} a_{n} \subset b \cap \bigcap_{n=1}^{\infty} \mathscr{G}_{n}
$$

At present we have no more significant results concerning $\zeta$-spaces, although some are presented in [9]. However in [7] M. Hochster shows that the class of spectral spaces of commutative rings coincides with the class of spectral spaces of distributive lattices; it follows that this correspondence carries over to minimal spectral spaces. In a paper "The minimal prime spectrum of a commutative ring" Hochster gives many deep results concerning the spaces of the title and by the above remark these all apply to the case of distributive lattices.

\section{Some basic homeomorphisms and isomorphisms}

The results in this section, apart from any intrinsic interest they may have, will serve to simplify certain proofs in later sections. Our first result is a direct analogue of one in [8] and has its proof omitted.

\section{Proposition 3.1. Minp $\mathbf{L} \cong \operatorname{Minp} \boldsymbol{\mu}_{\mathbf{L}}$.}

The map which effects the above homeomorphism is $M \rightarrow M \tau=\{\mathscr{M}(x): x \in M\}$. A similar result is the fact that the map $M \rightarrow M g=M \cap J$ where $J$ is an ideal of $\mathrm{L}$ is well-defined from $\mathscr{M}_{L}(J)$ into $\mathscr{M}_{J}$, and

\section{Proposition 3.2. $\mathscr{M}_{L}(J) \cong \operatorname{Minp} \mathbf{J}$.}

Proof. The fact that $\eta$ is well-defined and bijective follows exactly as in Proposition 1.2 of [14]. A routine argument will prove that corresponding basic open set map onto one another, giving the result that $\eta$ is continuous and open. For a similar situation see $\S 3$ of [14].

The set $\mathscr{M}_{\mathbf{L}}^{J}$ of all prime ideals which are minimal belonging to an ideal $J$ of $\mathbf{L}$ can be given a spectral topology exactly as $\mathscr{M}_{L}$ was (or, equivalently, the relative topology derived from $\operatorname{Spec} \mathbf{L}[14]$ ). We call the resulting space Minp ${ }^{J} \mathbf{L}$. The canonical homomorphism $\theta=\theta^{J}$ from $\mathbf{L}$ onto $\mathbf{L} / J$ induces an order isomorphism between $\mathscr{M}_{\boldsymbol{L}}^{J}$ and $\mathscr{M}_{\boldsymbol{L} / \boldsymbol{J}}$ and so we have, again omitting details, 
Proposition 3.3. $\operatorname{Minp}^{J} \mathbf{L} \cong \operatorname{Minp} \mathbf{L} / J$.

The special case $J=(x)^{*}$ for an element $x \in L$ is interesting, since we can show that an ideal $M \in \mathscr{M}^{(x)^{*}}$ is actually in $\mathscr{M}$. Thus

Proposition 3.4. $\mathscr{M}(x) \cong \operatorname{Minp} \mathrm{L} /(x)^{*}$.

Proof. Let $\xi$ be the map $M \rightarrow M \theta^{J}$ with $J=(x)^{*}$. Firstly $\xi$ maps $\mathscr{M}(x)$ into $\mathscr{M}_{L /(x)^{*}}$; if $M \in \mathscr{M}$ and $x \notin M$ then by $1.3(x)^{*} \subset M$ and so $M \in \mathscr{M}^{(x)^{*}}$ whence $M \theta \in \mathscr{M}_{\mathbf{L} /(\boldsymbol{x}) *}$. To prove that $\xi$ is bijective we will show that $\mathscr{M}(x)$ is exactly the set of all minimal prime ideals belonging to $(x)^{*}$; the latter it must be remembered are not, a priori, minimal prime ideals of $\mathbf{L}$.

Clearly $\mathscr{M}(x) \subset \mathscr{M}^{(x)^{*}}$. To prove the reverse inclusion take $P \in \mathscr{M}^{(x)^{*}}$, and for any $t \in P$ there is $t^{\prime} \notin P$ such that $t \wedge t^{\prime} \in(x)^{*}$ i.e. $t \wedge t^{\prime} \wedge x=0$. Now $x \notin P$, for if $x \in P$ it would contradict $P \supset(x)^{*}$. Thus for $t \in P$ we have $t^{\prime} \wedge x \notin P$ and $t \wedge\left(t^{\prime} \wedge x\right)=0$ whence $P$ is minimal prime belonging to zero.

Finally we omit a detailed proof of the homeomorphism.

Corresponding to the preceding topological equivalents we have some results relating the appropriate dual lattices; first however, we restate a result from [8]; recall that a lattice $\mathbf{L}$ with zero is disjunctive if to any pair $0 \neq a<b$ in $L$ there is $0 \neq c \in L$ such that $0=a \wedge c \neq b \wedge c$.

Proposition 3.5. The map $x \rightarrow \mathscr{M}(x)$ is a lattice epimorphism of $\mathbf{L}$ onto $\mu_{\mathbf{L}}$. It is an isomorphism iff $\mathbf{L}$ is disjunctive.

For $y \in L$ write $\mathscr{M}_{y}=\mathscr{M}_{(y)}$ for the set of minimal prime ideals of $(y)_{L}$. For any element $x \in(y)_{L}$ we can associate $\mathscr{M}_{y}(x)$ with $\mathscr{M}_{L}(x)$, and we find:

PROPOSITION 3.6. $\boldsymbol{\mu}_{(y)} \cong(\mathscr{M}(y))_{\mathfrak{p}}$.

The right-hand term is the principal ideal of $\mu_{L}$ generated by $\mathscr{M}_{(y)}$.

Proof. The map is easily checked to be an epimorphism so that we need only prove that it is injective. Suppose that for $x, x^{\prime}$ in $\mathscr{M}(y)$ we have $\mathscr{M}_{\mathbf{L}}(x)$ $=\mathscr{M}_{L}\left(x^{\prime}\right)$. Then, recalling that any $M_{1} \in \mathscr{M}_{y}(x)$ is of the form $M \cap(y)$ for some $M \in \mathscr{M}_{L}(y)$, we see that $M_{1} \in \mathscr{M}_{y}(x)$ means $M_{1}=M \cap(y)$ where $x \notin M$ and $y \notin M$, whence $x^{\prime} \notin M$ and so $M_{1} \in \mathscr{M}_{y}\left(x^{\prime}\right)$. The reverse inclusion follows similarly proving that $\mathscr{M}_{y}(x)=\mathscr{M}_{y}\left(x^{\prime}\right)$ and so completing the proof.

We close this section with some specialisations to annihilators. If for $y \in L$ we write $\theta$ or the canonical epimorphism of $\mathbf{L}$ onto $\mathbf{L} /(y)^{*}$, and keep the notation of the previous proposition, we have

PROPOSITION 3.7. $\boldsymbol{\mu}_{(y)} \cong \mu_{L /(y)^{*}}$

Proof. The map which effects the isomorphism is denoted by $\beta$ and given by $\mathscr{M}_{y}(x) \rightarrow \mathscr{M}_{\mathbf{L}^{\prime}(y)^{*}}(x \theta)$. We will only show that $\beta$ is injective; the fact that it is an epimorphism has its easy proof omitted. 
If

$$
\mathscr{M}_{L /(y) *}(x \theta)=\mathscr{M}_{\mathrm{L} /(y) *}\left(x^{\prime} \theta\right)
$$

for $x, x^{\prime}$ in $\mathscr{M}(y)$, we deduce that $\left(x:(y)^{*}\right)^{*}=\left(x^{\prime},(y)^{*}\right)^{*}$ and so, as in 3.4 that $\mathscr{M}_{L}(x)=\mathscr{M}_{L}\left(x^{\prime}\right)$ whence $\mathscr{M}_{y}(x)=\mathscr{M}_{y}\left(x^{\prime}\right)$ by 4.6. Thus the proof is completed.

COROLLARY 3.8. $\boldsymbol{\mu}_{(y)} \cong \boldsymbol{\mu}_{(y)^{* *}} \cong \boldsymbol{\mu}_{\boldsymbol{L} /(y)^{*}}$.

Proof. This follows from 3.7 and the fact that $\mathscr{M}(y)=\mathscr{M}\left((y)^{* *}\right) 1.3$ which implies that $\operatorname{Minp}(y) \cong \operatorname{Minp}(y)^{* *} \cong \mathscr{M}(y)$.

\section{Functorial properties}

In general the inverse image of a minimal prime ideal under a lattice morphism is prime but not necessarily minimal prime. (Consider the map $\alpha: 3 \rightarrow 2$ where $\mathbf{3}=\{0,1,2\}$ and $\mathbf{2}=\{0,1\}$ are the 3 - and 2-element chains, given by $0 \rightarrow 0$, $1 \rightarrow 0,2 \rightarrow 1$ ). Thus morphisms between lattices do not induce continuous functions between the corresponding $\operatorname{Minp}(\cdot)$ spaces except in certain special cases. The lattices which are of interest to us in this respect are Stone lattices: distributive pseudocomplemented lattices which satisfy $a^{*} \vee a^{* *}=1$ identically. For some properties of Stone lattices we refer to [13] and to [18]; in particular, in such lattices each prime ideal $P$ contains a unique minimal prime ideal which we write $(P)^{m}$. Under a (necessary) additional hypothesis this property characterises Stone lattices and so they appear to be the natural class of lattices to which the following proposition applies.

Proposition 4.1. Let $\phi: \mathbf{L}_{1} \rightarrow \mathbf{L}_{2}$ be a lattice epimorphism between two Stone lattices. Then

$$
{ }^{b} \phi: \mathscr{M}_{L_{2}} \rightarrow \mathscr{M}_{L_{1}}
$$

given by $M^{b} \phi=\left(M \phi^{-1}\right)^{m}$ defines a continuous function between $M$ inp $\mathbf{L}_{2}$ and $\operatorname{Minp} \mathrm{L}_{1}$.

Proof. Firstly ${ }^{b} \phi$ is well defined since $M \phi^{-1}$ is prime and so we use the remark above. Also ${ }^{b} \phi$ is continuous since for an ideal $J$ of $\mathbf{L}_{1}$ we have

$$
\mathscr{M}_{\boldsymbol{L}_{1}}(J)\left({ }^{b} \phi\right)^{-1}=\mathscr{M}_{\boldsymbol{L}_{2}}(J \phi)
$$

and so the inverse image of an arbitrary open set is open. This completes the proof.

It is not hard to prove that the correspondence $\mathbf{L} \rightarrow \operatorname{Minp} \mathbf{L}$ is functorial between the category of Stone lattices (as abstract algebras) and Boolean spaces. Indeed this contravariant functor sends arbitrary products (coproducts) into coproducts (products).

\section{Compactness}

As noted in [6] compactness in spaces of ideals is traditionally associated with the presence of an identity, but not in the case of minimal prime ideals. 
What is more important in this situation is a certain property of annihilators. However in the lattice (resp. commutative l-group, commutative ring) context the existence of dense elements (resp. weak order units, non-divisors of zero) is necessary but not sufficient for compactness; these are "almost" identities and so some harmony with the traditional situation is preserved.

In our works [12] and [15] we have presented some results on this topic which we now repeat before extending them. See 1.2 for the definiticn of $D=D_{L}$.

Proposirion 5.1. Let $\mathbf{L}$ be a distributive lattice with zero. Then the following are equivalent:

(i) For any $x \in L$ there exists $x^{\prime} \in L:(x)^{* *}=\left(x^{\prime}\right)^{*}$.

(ii) $\operatorname{Minp} \mathbf{L}=\operatorname{Min} p \mathbf{L}$.

(iii) Minp $\mathbf{L}$ is compact.

(iv) $\mu_{L}$ is a Boolean lattice.

(v) For any $x \in L$ there exists $x^{\prime} \in L: x \wedge x^{\prime}=0, x \vee x^{\prime} \in D$.

(vi) For any ideal $J$ with $J \cap D=\phi$, there is $M \in \mathscr{M}_{L}: J \subset M$.

(vii) $\{h(x): x \in L\}$ is a basis for the open sets of Minp $\mathbf{L}$.

(viii) $\{\mathscr{M}(x): x \in L\}$ is a basis for the open sets of Minp $\mathbf{L}$.

Proof. (i), (ii), (iii), (iv)), (v), (vi) are proved equivalent in [12] while (vii) and (viii) appear (in the case of a commutative semigroup) with (iii) in [15].

We now ask when the basic open sets $\mathscr{M}(y)$ are compact, leading up to some conditions saying when Minp $L$ is locally compact; we begin with a purely algebraic result.

Proposition 5.2. For $y \in L$ the following are equivalent:

(i) For any $x \in L$ there is $x^{\prime} \in L$ :

$$
\left(\left(x:(y)^{*}\right)^{*}:(y)^{*}\right)^{*}=\left(x^{\prime}:(y)^{*}\right)^{*} .
$$

(ii) For any $x \in L$ there is $x^{\prime} \in L$ :

$$
x \wedge x^{\prime} \in(y)^{*}, x \vee x^{\prime} \in D^{(y)^{*}} .
$$

(iii) For any $t \in(y)^{* *}$ there is $t^{\prime} \in(y)^{* *}$ :

$$
t \wedge t^{\prime}=0, t \vee t^{\prime} \in D_{(y)^{* *}}
$$

Proof. (i) implies (ii). We show that the $x^{\prime}$ of (i) satisfies the condition of (ii).

Firstly, $x \wedge x^{\prime} \in(y)^{*}$ since $x \in\left(\left(x:(y)^{*}\right)^{*}:(y)^{*}\right)^{*}$ and so $x \in\left(x^{\prime}:(y)^{*}\right)^{*}$. Further, if $t \wedge\left(x \vee x^{\prime}\right) \in(y)^{*}$ we must have $t \in\left(x^{\prime}:(y)^{*}\right)^{*}$, and $t \in\left(x:(y)^{*}\right)^{*}$. But this means that $\left.t \in\left(\left(x:(y)^{*}\right)^{*}\right)^{*}:(y)^{*}\right)^{*}$ and so $t \wedge t \in(y)^{*}$. This proves that $x \vee x^{\prime} \in D^{(y)^{*}}$.

(ii) implies (iii). Take $t \in(y)^{* *}$ and consider it is an element of $L$. There is $t^{\prime}$ by (ii) such that $t \wedge t^{\prime} \in(y)^{*}$ and $t \vee t^{\prime} \in D^{(y)^{*}}$. Now since $t \in(y)^{* *}$ we have 
$t \wedge t \wedge t^{\prime}=0$ i.e. $t \wedge t^{\prime}=0$. Further, if $s \wedge\left(t \vee t^{\prime}\right)=0$ for $s \in(y)^{* *}$ we have $s \in(y)^{*}$ and so $s \in(y)^{* *} \cap(y)^{*}$.

Thus $t \vee t^{\prime}$ is dense in $(y)^{* *}$ and so $t \vee t^{\prime} \in D_{(y) * *}$.

(iii) impies (i). Take $x \in L$ and let $x^{\prime}=(x \wedge y)^{\prime}$ where $x \wedge y \in(y)^{* *}$ and so $(x \wedge y)^{\prime}$ is defined and has the appropriate properties by (iii).

Then $x \wedge y \wedge x^{\prime}=(x \wedge y) \wedge(x \wedge y)^{\prime}=0$ and so $x \wedge x^{\prime} \in(y)^{*}$, giving $x \in\left(x^{\prime}:(y)^{*}\right)^{*}$. The reverse inclusion follows by using the fact that $(x \wedge y) \vee(x \wedge y)^{\prime}$ is dense in $(y)^{* *}$.

We omit the details.

The next result is part algebraic, part topological. The conditions in it will shortly be seen to be equivalent to those in 5.2.

Proposition 5.3. For any $y \in L$ the following are equivalent:

(i) $\mathscr{M}(y)$ is compact as a subset of $\operatorname{Minp} \mathbf{L}$.

(ii) For any $x \in L$ there is $x^{\prime} \in L: \mathscr{M}(y) \cap h(x)=\mathscr{M}\left(y \wedge x^{\prime}\right)$.

(iii) $(\mathscr{M}(y))_{\boldsymbol{\mu}}$ is a Boolean sublattice of $\boldsymbol{\mu}_{\boldsymbol{L}}$.

Proof. (i) implies (ii). Assume $\mathscr{M}(y)$ is compact. Then for any $x \in L$, $\mathscr{M}(y) \cap h(x) \cap \bigcap\left\{h(t): t \in\left(x:(y)^{*}\right)^{*}\right\}=\phi$. This readily is checked. Compactness implies $\mathscr{M}(y) \cap h(x) \cap \bigcap\left\{h\left(t_{i}\right): t_{i} \in\left(x:(y)^{*}\right)^{*}, \quad i=1,2, \cdots, n\right\}=\phi$, or $\mathscr{M}(y) \cap h(x) \cap h\left(x^{\prime}\right)=\phi$ where $x^{\prime}=V_{i} t_{i} \in\left(x:(y)^{*}\right)^{*}$.

We will prove $\mathscr{M}(y) \cap h(x)=\mathscr{M}\left(y \wedge x^{\prime}\right)=h\left(\left(y \wedge x^{\prime}\right)^{*}\right)$.

Suppose $M \in \mathscr{M}(y) \cap h(x)$ and $t \in L$ is such that $t \wedge y \wedge x^{\prime}=0$. Then since $x^{\prime} \notin M$ by the above equation $\left(^{*}\right), y \wedge x^{\prime} \notin M$ and so $t \in M$. Thus $\left(y \wedge x^{\prime}\right)^{*} \subset M$ and so $M \in \mathscr{M}\left(y \wedge x^{\prime}\right)$.

Conversely, if $M \in \mathscr{M}\left(y \wedge x^{\prime}\right)$ then $y \notin M$ and $x^{\prime} \notin M$ and so $x \notin M$ since $\mathrm{x} \wedge x^{\prime} \wedge y=0$. Thus $M \in \mathscr{M}(y) \cap h(x)$. The implication is proved.

(ii) implies (iii). For any $\mathscr{M}(t) \in(\mathscr{M}(y))_{\mu}$ we have $\mathscr{M}(t) \subset \mathscr{M}(y)$ and so $\mathscr{M}(t)=\mathscr{M}(t \wedge y)$. By (ii), there is $t^{\prime} \in L$ such that $\mathscr{M}(y) \cap h(t \wedge y)=\mathscr{M}\left(y \wedge t^{\prime}\right)$. Now we have $\mathscr{M}(y \wedge t) \cap \mathscr{M}\left(y \wedge t^{\prime}\right)=\phi$ and $\mathscr{M}(y \wedge t) \cup \mathscr{M}\left(y \wedge t^{\prime}\right)=\mathscr{M}(y)$ and so $(\mathscr{M}(y))_{\mu}$ is a Boolean as a sublattice of $\boldsymbol{\mu}_{\boldsymbol{L}}$.

(iii) implies (i). Suppose we have a family of closed sets in $\mathscr{M}(y)$ which have the finite intersection property. Then by (iii) they are of the form $\{\mathscr{M}(t)\}$ with $\mathscr{M}(t) \in(\mathscr{M}(y))$ and $\bigcap\{\mathscr{M}(t): t \in \mathbf{u}\} \neq \phi$ for all finite $\mathbf{u} \subset T$. Exactly as in 2.4 we prove that there is a minimal prime ideal $M$ with $M \in \bigcap\{\mathscr{M}(t): t \in T\}$ and so $\mathscr{M}(y)$ is compact.

PRoposition 5.4. For $y \in L$ the following are equivalent:

(i) For any $x \in L$ there exists $x^{\prime} \in L$ such that

$$
x \wedge x^{\prime} \in(y)^{*}, x \vee x^{\prime} \in D^{(y)^{*}} .
$$

(ii) For any $x \in L$ there exists $x^{\prime} \in L$ such that

$$
\mathscr{M}(y) \cap h(x)=\mathscr{M}\left(y \wedge x^{\prime}\right) .
$$


Proof. (i) implies (ii). Choose the $x^{\prime}$ in (ii) to be the one given by (i).

Now if $M \in \mathscr{M}(y) \cap h(x), y \notin M$ and so $(y)^{*} \subset M$ and $x \in M$. We must prove that $x^{\prime} \notin M$. If $x^{\prime} \in M$ then $x \vee x^{\prime} \in M$ would follow proving that $\left(x \vee x^{\prime}\right) \wedge s=0$ for $s \notin M$. But $s \wedge\left(x \vee x^{\prime}\right)=0 \in(y)^{*}$ implies $s \in(y)^{*}$ which contradicts $(y)^{*} \subset M$. Thus

$$
\mathscr{M}(y) \cap h(x) \subset \mathscr{M}\left(y \wedge x^{\prime}\right) .
$$

For the reverse inclusion, if $y \wedge x^{\prime} \notin M$ then $y \notin M$ and, since $y \wedge x^{\prime} \wedge x=0 \in M$, we must have $x \in M$. This proves the reverse inclusion and so $\mathscr{M}(y) \cap h(x)$ $=\mathscr{M}\left(y \wedge x^{\prime}\right)$.

(ii) implies (i). Again we show that the $x^{\prime}$ defined by (ii) satisfies the conditions of (i). Firstly, $x \wedge x^{\prime} \in(y)^{*}$ since for any $M \in \mathscr{M}(y)$ either $x \in M$ or $x^{\prime} \in M$ (by $\left.\mathscr{M}(y) \cap h(x)=\mathscr{M}\left(y \wedge x^{\prime}\right)\right)$ and so $x \wedge x^{\prime} \in(y)^{*}=k(\mathscr{M}(y))$.

Also, if $s \wedge\left(x \vee x^{\prime}\right) \in(y)^{*}, s \wedge x \wedge y=0$ and $s \wedge x^{\prime} \wedge y=0$.

Then $s \in M$ for any $M \in \mathscr{M}\left(y \wedge x^{\prime}\right)=\mathscr{M}(y) \cap h(x)$.

Also $s \in M$ for any $M \in \mathscr{M}(y) \backslash \mathscr{M}(y) \cap h(x)$.

Thus $s \in(y)^{*}=k(\mathscr{M}(y))$ and so $x \wedge x^{\prime} \in D^{(y)^{*}}$.

Proposition 5.5. For any $y \in L$ the following are equivalent:

(i) For any $x \in L$ there is $x^{\prime} \in L$ :

$$
\left(\left(x:(y)^{*}\right)^{*}:(y)^{*}\right)^{*}=\left(x^{\prime}:(y)^{*}\right)^{*} .
$$

(ii) For any $x \in L$ there is $x^{\prime} \in L$ :

$$
x \wedge x^{\prime} \in(y)^{*}, x \vee x^{\prime} \in D^{(y)^{*}} .
$$

(iii) For any $t \in(y)^{* *}$ there is $t^{\prime} \in(y)^{* *}$ :

$$
t \wedge t^{\prime}=0, t \vee t^{\prime} \in D_{(y) * *}
$$

(iv) $\mathscr{M}(y)$ is a compact subset of Minp $\mathbf{L}$.

(v) For any $x \in L$ there is $x^{\prime} \in L$ :

$$
\mathscr{M}(y) \cap h(x)=\mathscr{M}\left(y \vee x^{\prime}\right) .
$$

(vi) $(\mathscr{M}(y))_{\mu}$ is Boolean as a sublattice of $\mu_{\boldsymbol{L}}$.

Proof. (i), (ii) and (iii) are equivalent by 5.2;

(iv), (v) and (vi) are equivalent by 5.3 ;

(ii) and (v) are equivalent by 5.4 .

A brief condition concerning the non-compactness of $\mathscr{M}(y)$ is the following:

Proposition 5.6. For $y \in L$, if the subset $\mathscr{M}(y)$ of Minp $\mathbf{L}$ is not compact, there is an ideal $J$ of $\mathbf{L}$ with $J^{*} \subset(y)^{*}$ but $(j)^{*} \notin(y)^{*}$ for any $j \in J$.

ProOF. If $\mathscr{M}(y)$ is not compact, there is a set $\{\mathscr{M}(t): t \in T\}$ with $\bigcup\{\mathscr{M}(t) ; t \in T\} \supset \mathscr{M}(y)$ but $\bigcup\{\mathscr{M}(t): t \in \mathbf{u}\} \neq \mathscr{M}(y)$ for any finite $\mathbf{u} \subset T$. Take 
$J=(T)$, and we have $\mathscr{M}(J) \supset \mathscr{M}(y)$ or $J^{*} \subset(y)^{*}$, but $\mathscr{M}(j) \neq \mathscr{M}(y)$ or $(j)^{*} \phi(y)^{*}$ for any $j \in J$. These last results follow on taking $k(\cdot)$ of both sides of the appropriate relation.

Before proving our main result concerning local compactness of Minp $\mathbf{L}$ a preliminary proposition is needed.

Proposition 5.7. Define $K \subset L$ by $K=\{x \in L: \mathscr{M}(x)$ is compact in MinpL $\}$. Then $K$ is an ideal of $\mathbf{L}$ and $\{\mathscr{M}(x): x \in K\}$ is relatively complemented as $a$ sublattice of $\mu_{\mathbf{L}}$.

Proof. If $x, y \in K$ then $\mathscr{M}(x) \cup \mathscr{M}(y)$ is compact and so $x \vee y \in K$. Also if $x \in K$ and $t \leqq x$ then $t \in K$ since $\mathscr{M}(t) \subset \mathscr{M}(x)$ and a closed subset of a compact set is compact. Thus $K$ is an ideal. Regarding the last asertion, 5.5 tells us that $(\mathscr{M}(x))_{\mathfrak{u}}$ is complemeneted for $x \in K$ and the result follows.

Proposition 5.8. The following are equivalent:

(i) Minp $\mathbf{L}$ is locally compact.

(ii) For any $M \in \mathscr{M}$ there is $x \in M: \mathscr{M}(x)$ is compact in $M$ inp $\mathbf{L}$.

(iii) $\mathscr{M}(K)=\mathscr{M}$.

(iv) $\{\mathscr{M}(x): x \in K\}$ join-generates $\mu_{L}$.

Proof. (i) implies (ii). Suppose MinpL is locally compact. Then for every $M \in \mathscr{M}$ there is a compact neighbourhood $\mathscr{C}$ say with $M \in \mathscr{C}$. Now for any neighbourhood $\mathscr{C}$ of $M$ there is a basis set $\mathscr{M}(x) \subset \mathscr{M}$ with $M \in \mathscr{M}(x)$. Such an $\mathscr{M}(x)$ must be compact and the implication is proved.

(ii) implies (iii). Assuming (ii) we have: for any $M \in \mathscr{M}$ there is $x \notin M$ with $x \in K$. This implies $\mathscr{M}(K)=\mathscr{M}$ as required.

(iii) implies (iv). We must prove that for any $y \in L$ there is a subset $K_{y} \subset K$ with $\mathscr{M}(y)=\bigcup\left\{\mathscr{M}(x): x \in K_{y}\right\}$.

Assume $\mathscr{M}(K)=\mathscr{M}$ and we see that

$$
\begin{aligned}
\mathscr{H}(y) & =\mathscr{M}(y) \cap \mathscr{M}(K)=\mathscr{M}((y) \cap K) \\
& =\bigcup\{\mathscr{M}(x): x \in(y) \cap K\} .
\end{aligned}
$$

This proves the assertion.

(iv) implies (i). Since every set in the open basis is a union of compact sets (by (iv)) every point $M \in \mathscr{M}$ has a compact neighbourhood and so $\mathbf{M i n p} \mathbf{L}$ is locally compact.

Corollary 5.9. If $\mu_{L}$ is a generalised Boolean lattice, then Minp $L$ is locally compact.

Proof. In this situation, every $(\mathscr{M}(y))_{\mu}$ would be Boolean and so, by 5.5 , each $\mathscr{M}(y)$ would be compact. 
Now it might be hoped that the following extension of 5.1 could be proved: Minp $\mathbf{L}$ is locally compact iff $\mu_{\boldsymbol{L}}$ is a generalised Boolean lattice. But this is not so as is shown by J. H. Rubinstein in [9]. One final result before mentioning a compactification of $\operatorname{Minp} \mathbf{L}$ is:

Proposition 5.10. Suppose that $\mathbf{L}$ is closed under countable joins and satiskes

$$
x \wedge \vee\left\{y_{i}: i \in I\right\}=\vee\left\{x \wedge y_{i}: i \in I\right\}
$$

for I a countable set. Then Minp $\mathbf{L}$ is countably compact.

Proof. Suppose $\left\{M_{n}: n \in H\right\}$ is a sequence of minimal prime ideals of $L$. Take $\mathscr{U}$ a free ultrafilter on $N$ and define $M(\mathscr{U})=\left\{x \in L:\left\{n \in N: x \in M_{n}\right\} \in \mathscr{U}\right\}$. It is a prime ideal and we show that it is minimal. Take $x \in M(\mathscr{U})$ and $\left\{n: x \in M_{n}\right\} \in \mathscr{U}$. From $x \in M_{n}$ we deduce that there is $y_{n} \notin M_{n}$ with $x \wedge y_{n}=0$. For $y=\vee\left\{y_{n}: x \in M_{n}\right\}$ we find that $y \notin M(\mathscr{U})$ and using the distributivity condition, $x \wedge y=0$.

The fact that $M(\mathscr{U})$ is a cluster point of the sequence follows exactly as in [14].

And now, a compactification of Minp $L$. Form the Boolean lattice $\boldsymbol{\beta}_{\boldsymbol{L}}$ $=\left(\beta_{L} ; \cup, \cap^{\prime}, \phi\right)$ generated by $\{\mathscr{M}(x): x \in L\}=\mu_{L}$ and then let $X$ be the set of prime (= maximal) ideals of $\beta_{L}$. Then the map $\tau: \mathscr{M} \rightarrow X$ given by $M \tau$ $=\left\{D \in \beta_{L}: M \notin D\right\}$ is readily checked to define a homeomorphism of Minp $\mathbf{L}$ onto a dense subspace of the space of prime ideals of $\beta_{L}$, which is compact.

\section{Connectedness}

The space Minp $\mathbf{L}$ can exhibit many extreme forms of disconnectedness. We begin with:

Proposition 6.1. Minp $\mathbf{L}$ is totally disconnected.

Proof. This is immediate from 2.6, see [2, Part 2] p. 200.

A subset of a topological space is called a $G_{\delta}$ if it is the intersection of a sequence of open sets. The complement of a $G_{\delta}$ is called an $F_{\sigma}$.

Lemma 6.2. Every compact $G_{\delta}$ in Minp $\mathbf{L}$ is expressible as a countable intersection of the sets $\mathscr{M}(x)(x \in L)$.

Proof. Let $Y$ be a compact $G_{\delta}$ i.e. $Y=\bigcap_{n} A_{n}$ where each $A_{n}$ is open in Minp L. Then $A_{n}=\bigcup\left\{\mathscr{M}(a): a \in B_{n}\right\}$ for some $B_{n} \subset L$ and so $Y=\bigcap_{n} A_{n} \subset A$ $=\bigcup\left\{\mathscr{M}(a): a \in B_{n}\right\}$ whence, by compactness of $Y$, we deduce that $Y \subset \mathscr{M}\left(b_{n}\right) \subset A_{n}$ for some $b_{n} \in L$. Thus $Y=\bigcap_{n} \mathscr{M}\left(b_{n}\right)$ and the proof is complete.

Although the next result will soon be generalised it is stated separately because of its special interest. First we need some definitions: a subset $Z$ of 
topological space $X$ is called a zero-set ([5] p. 14) if there exists a continuous real-valued function $f$ defined on $X$ such that $Z=\{x \in X: f(x)=0\}$. It is shown in [5] p. 15 that every zero-set is a $G_{\delta}$. A subset $W$ of a topological space $X$ is called a cozero-set if $X \backslash W$ is a zero-set, and $X$ is called basically disconnected if every cozero-set in $X$ has open closure. We refer to [5] where many properties of this class of topological spaces are given. We use $\sigma$ to denote $\aleph_{0}$ in order to be consistent with normal usage in Boolean algebras.

Proposition 6.3. The following are equivalent:

(i) Condition $\sigma\left({ }^{*}\right)$ : For any $A \subset L$ with $|A| \leqq \sigma$ there is $a^{\prime} \in L: A^{* *}=\left(a^{\prime}\right)^{*}$.

(ii) $\boldsymbol{\mu}_{\mathbf{L}}$ is a $\sigma$-complete Boolean lattice.

(iii) Minp $\mathrm{L}$ is compact and basically disconnected.

Proof. (i) and (ii) are proved equivalent in Theorem 2 of [11].

(ii) implies (iii). Firstly $\boldsymbol{\mu}_{\boldsymbol{L}}$ is Boolean and so by $5.1 \operatorname{Minp} \mathbf{L}$ is compact. Take a zero-set $Z$; by a preceding remark and the compactness of $\operatorname{Minp} \mathbf{L} Z$ is a compact $G_{\delta}$ and so by 6.2 above, expressible in the form $Z=\bigcap_{n} \mathscr{M}\left(b_{n}\right)$. Again by the compactness of $\operatorname{Minp} \mathbf{L}$, each $\mathscr{M}\left(b_{n}\right)$ is equal to $h\left(b_{n}^{\prime}\right)$ for some $b_{n}^{\prime}$, and so $\mathscr{M} \backslash Z=\bigcup_{n} \mathscr{M}\left(b_{n}^{\prime}\right)$. Also 4.1 implies that $\operatorname{Minp} \mathbf{L}$ is the Boolean representation space of the $\sigma$-complete Boolean lattice $\boldsymbol{\mu}_{\boldsymbol{L}}$, and so known results imply that $\bigcup_{n} \mathscr{M}\left(b_{n}^{\prime}\right)$ has open closure: see $[10] \S 22.4$. This proves that complements of zerosets have open closure, i.e. Minp $\mathbf{L}$ is basically disconnected.

(iii) implies (ii). Let $A \subset L$ be a countable set. Then $\mathscr{U}=\bigcup\{\mathscr{M}(a): a \in A\}$ is an open $F_{\sigma}$ in a normal space and so a co-zero set. Thus

$$
\begin{aligned}
\mathscr{U}^{-} & =(\bigcup\{\mathscr{M}(a): a \in A\})^{-} \\
& =h(\cap\{k(\mathscr{M}(a)): a \in A\})=h\left(A^{*}\right)
\end{aligned}
$$

is open-closed. By the assumed compactness of Minp $\mathbf{L}$ and by 5.1 there is $a^{\prime} \in L$ such that $\mathscr{U}^{-}=h\left(A^{*}\right)=h\left(a^{\prime}\right)$. But then $A^{* *}=\left(a^{\prime}\right)^{*}$ and our proof is complete.

We now present the generalisation of 6.3 referred to above. In the following m denotes a cardinal larger than 1 .

Proposition 6.4. The following are equivalent:

(i) Condition $\mathbf{m}\left({ }^{*}\right)$ : For any $A \subset L$ with $|A| \leqq m$ there is $a^{\prime} \in L: A^{* *}=\left(a^{\prime}\right)^{*}$.

(ii) Minp $\mathbf{L}$ is compact and the union of not more than $\mathbf{m}$ open-closed subsets of Minp L has open closure.

(iii) $\boldsymbol{\mu}_{\boldsymbol{L}}$ is an $\mathbf{m}$-complete Boolean lattice.

Proof. (i) and (ii) are equivalent by Theorem 2 of [11] Since Minp $L$ is also (canonically) the Boolean representation space of $\mu_{L}$ when (ii) or (iii) are assumed, (ii) and (iii) are equivalently by known results concerning Boolean lattices [10] $\S 22.4$. 
Corollary 6.5. If $\mathbf{L}$ is closed under m-ary joins and is pseudocomplemented, then the properties of 6.4 follow.

Proof. For $A \subset L$ with $|A| \leqq m$ write $a=\vee A$. Then $(a)^{*}=\left(a^{*}\right)$ where $a^{*}$ is the pseudocomplement of $a$, and so $A^{* *}=(a)^{* *}=\left(a^{*}\right)^{*}$. Thus 6.4 (i) holds and so the rest follow.

A topological space $X$ is called extremally disconnected if the closure of every open set is open - see [5] for some results on this class of spaces.

COROLlary 6.6. The following are equivalent:

(i) For any $A \subset L$ there is $a^{\prime} \in L: A^{* *}=\left(a^{\prime}\right)^{*}$.

(ii) Minp $\mathbf{L}$ is compact and extremally disconnected.

(iii) $\boldsymbol{\mu}_{\boldsymbol{L}}$ is a complete Boolean lattice.

Proof. This is just 6.4 for all cardinals $\mathbf{m}$ however large.

We next push the main idea a little further making use of the homeomorphism $\operatorname{Minp}(y)^{* *} \cong \mathscr{M}_{\mathbf{L}}(y)\left(3.2\right.$ with $\left.J=(y)^{* *}\right)$ and the isomorphism $\boldsymbol{\mu}_{(y) * *} \cong(\mathscr{M}(y))_{\mu}$ (3.8).

Proposition 6.7. The following are equivalent:

(i) For all $y \in L,(y)^{* *}$ satsifies condition $\sigma\left(^{*}\right)$ of 6.3 .

(ii) $\boldsymbol{\mu}_{L}$ is a conditionally $\sigma$-complete generalised Boolean lattice.

(iii) For all $y \in L \mathscr{M}(y)$ is compact in Minp $\mathbf{L}$, and Minp $\mathbf{L}$ is basically disconnected.

Proof. (i) implies (ii). Assuming (i) we deduce using 3.8 and 6.3 (ii) that $\boldsymbol{\mu}_{(\boldsymbol{y}) * *}$ is a $\sigma$-complete Boolean lattice or all $y \in L$, i.e. (ii) above.

(ii) implies (ii). By the implication (ii) implies (iii) of 6.3 and 3.2, we deduce that each set $\mathscr{M}(y)$ is compact and basically disconnected. Our conclusion now follows from the (topological) lemma 4.6 of [6], i.e. if all the basic sets $\mathscr{M}(y)$ are basically disconnected, then $\operatorname{Minp} L$ is basically disconnected.

(iii) implies (i). Assuming that all the $\mathscr{M}(y)$ are compact and basically disconnected (which is immediate from (iii)) we use 3.2, 3.8 and the implication (iii) implies (i) of 6.3 to complete the proof.

The final two results follow from 6.4 and 6.6 using 3.2 and 3.8 exactly as 6.7 followed from 6.3

Proposition 6.8. The following are equivalent:

(i) For all $y \in L,(y)^{* *}$ satisfies condition $m\left({ }^{*}\right)$ of 6.4 .

(ii) $\boldsymbol{\mu}_{\mathbf{L}}$ is a conditionally $\mathbf{m}$-complete generalised Boolean lattice.

(iii) For all $y \in L \mathscr{M}(y)$ is compact in Minp $\mathbf{L}$, and the union of not more than $\mathbf{m}$ open-closed subsets of Minp $\mathrm{L}$ has open closure. 
Proposition 6.9. The following are equivalent:

(i) For any $y \in L,(y)^{* *}$ satisfies: for any $A \subset(y)^{* *}$ there exists $a^{\prime} \in(y)^{* *}$ : $A^{* *}=\left(a^{\prime}\right)^{*}$.

(ii) $\boldsymbol{\mu}_{\boldsymbol{L}}$ is a conditionally complete generalised Boolean lattice.

(iii) For all $y \in L \mathscr{M}(y)$ is compact in Minp $\mathbf{L}$, and Minp $\mathbf{L}$ is extremally disconnected.

\section{Examples and counterexamples}

In general $t$ does not seem easy to explicitly describe $\operatorname{Minp} \mathbf{L}$ for a given $\mathbf{L}$, at least not for many lattices which arise naturally. Exceptions, of course, are lattices where $\operatorname{Minp} \mathbf{L}$ is a compact space and the problem reduces to the theory of Boolean spaces. A possible explanation of this fact is that there is no body of knowledge built up about spaces of prime (or maximal) ideals of lattices which is comparable to that which exists regarding commutative rings or commutative Banach algebras.

What we can do sometimes is relate the space Minp $\mathbf{L}$ to some other space about which much more is known, or more often obtain a lattice $\mathbf{L}$ from an object associated with a space about which we have knowledge, and thereby deduce information about Minp L. It is this approach, similar to looking for problems for which one has a solution, which we take below.

\section{A. The Lattices $\mathbf{L}(\mathbf{X})$.}

In this subsection $X$ will always denote a completely regular topological space, [5] p. 36. We have already defined a zero-set in a topological space, however we introduce the notation (c.f. [5] Chap. 1)

$$
\mathbf{Z}(f)=\{x \in X: f(x)=0\}
$$

where $f \in C(X)$, the set of all continuous real-valued functions defined on $X$. It is not hard to see that $\mathbf{Z}(f g)=\mathbf{Z}(f) \cup \mathbf{Z}(g)$ and $\mathbf{Z}(|f|+|g|)=\mathbf{Z}(f) \cap \mathbf{Z}(g)$ where $f, g \in C(X)$, and so the family $\mathbf{Z}(X)=\{\mathbf{Z}(f): f \in C(X)\}$ forms a distributive lattice called the lattice of zero-sets of $X$. These definitions apply to any topological space, but for later use we need the following:

LeMMA 7.1. If $X$ is completely regular, then $\mathrm{Z}(X)$ is disjunctive.

Proof. Suppose $\phi \neq \mathbf{Z}(f) \underset{\neq}{\subset} \mathbf{Z}(g)$. Then choose $x_{0} \in \mathbf{Z}(g) \backslash \mathbf{Z}(f)$ and, by the definition of complete regularity, there is a continuous function $h$ defined on $X$ such that $h(x)=0$ for all $x$ in the closed set $\mathbf{Z}(f)$, and $h\left(x_{0}\right)=1$ for the point $x_{0} \notin \mathbf{Z}(f)$. Clearly $\mathbf{Z}(1-h) \cap \mathbf{Z}(f)=\phi$ and $\mathbf{Z}(1-h) \cap \mathbf{Z}(g) \neq \phi$; thus $\mathbf{Z}(X)$ is disjunctive.

Our next result provides a useful means of identifying $\mathscr{M}_{\mathbf{z}(\boldsymbol{X})}$. 
Lemma 7.2. Minp $\mathrm{Z}(X)$ and $\beta X$ (the Stone-Cech compactification of $X$ ) are canonically homeomorphic.

Proof. We use the definition of $\beta X$ given in [5] pp. $86-87$ i.e. $\beta X$ is the set of all $z$-ultrafilters on $X$ equipped with a certain topology. Now it follows from the definitions in [5] (which we omit) that a $z$-ultrafilter on $X$ is just a maximal dual ideal of the lattice $\mathbf{Z}(X)$, and so the set-theoretic complement (in $\mathbf{Z}(X)$ ) of a minimal prime ideal of $\mathbf{Z}(X)$. The topology which the set of all $z$-ultrafilters is given is easily seen to carry over, via the correspondence just mentioned, to the dual spectral topology on $\mathscr{M}_{Z(X)}$ and so the proof is complete.

By the preceding Lemma it is clear that $\operatorname{Minp} \mathbf{Z}(X)$ will equal $\beta X$ whenever $\mathbf{Z}(X)$ satisfies any of the conditions of 5.1 ; in fact this is very rarely. A topological space $X$ is called a $P$-space if every zero set is open - this is equivalent to the definition given in [5] where further facts about $P$-spaces may be found.

PROPOSITION 7.3. The following are equivalent:

(i) $\operatorname{Minp} \mathbf{Z}(X)$ is compact.

(ii) Minp $\mathbf{Z}(X)$ is canonically homeomorphic with $\beta X$.

(iii) $\mathrm{Z}(X)$ is a Boolean lattice.

(iv) $X$ is a $P$-space.

Proof. (i) and (ii) are equivalent by 5.1 and 7.1.

(i) and (iii) are equivalent by Theorem 2 of [12] in the light of 7.2 above.

Also since zero-sets are closed (iii) implies (iv) and (iv) similarly implies (iii), completing the proof.

Thus any completely regular topological space $X$ which is not a $P$-space (e.g. an infinite compact space) gives us a lattice $\mathbf{Z}(X)$ for which $\operatorname{Minp} \mathbf{Z}(X)$ is non-compact. In fact we have more. Recall that a space $X$ is perfectly normal if every closed set is a $G_{\delta}$.

Proposition 7.4. Let $X$ be a perfectly normal compact space. Then $\operatorname{Minp} \mathrm{Z}(X)$ is homeomorphic to the set $X$ equipped with the discrete topology.

Proof. In this case $\mathbf{Z}(X)$ is just the lattice of all closed subsets of $X$. We assert that for $x \in X$ the set $M_{x}=\{a \in Z(X): x \notin a\}$ is a minimal prime ideal of $\mathbf{Z}(X)$, and that all minimal primes of $\mathbf{Z}(X)$ are of this form. Clearly $M_{x}$ is a prime ideal; for $a \in M_{x}$ we have $x \notin a$ and by the normality of $X$ we can find a closed $b \supset\{x\}$ with $a \cap b=\phi$ i.e. $b \notin M_{x}$ ond $a \cap b=\phi$, which characterises $M_{x}$ as a minimal prime ideal. Next take a minimal prime $M$ of $\mathbf{Z}(X)$; the family $\mathbf{Z}(X) \backslash M$ has the f.i.p. and so, by compactness $\cap \mathbf{Z}(X) \mid M \neq \phi$. It is easy to see that this intersection can only contain a single point, say $x$, and then $M=M_{x}$.

Thus $X$ and $\mathscr{M}_{\mathrm{Z}(X)}$ correspond bijectively, and we now turn to describe the topology on $\mathscr{M}_{\mathrm{Z}(X)}$. Let $a \in \mathbf{Z}(X)$ be a closed set; then $\mathscr{M}(a)=\left\{M \in \mathscr{M}_{\boldsymbol{Z}(X)}\right.$ : 
$a \notin M\}$ is open by definition, i.e. $\left\{M_{x}: x \in X\right.$ and $\left.x \in a\right\}$ is open. In particular each set $\left\{M_{x}\right\}$ is open and thus the topology on $\mathscr{M}_{\mathbf{z}(X)}$ is discrete completing our proof.

In later work we will just identify $\operatorname{Minp} \mathbf{Z}(X)$ with $X$ when $X$ is a perfectly normal compact space.

\section{B. The Lattices $\mathrm{L}(\mathrm{A})$}

In this section $\mathbf{A}$ will denote a commutative ring, not necessarily with identity; we will find that for any such $\mathbf{A}$ there is a lattice $\mathbf{L}(\mathbf{A})$ with canonically homeomorphic space of minimal prime ideals. The space $X=\operatorname{Spec} \mathbf{A}$ of the ring $\mathbf{A}$ is fully discussed in [3], [7], and the corresponding space $\operatorname{Spec} \mathbf{L}$ for $\mathbf{L}$ a distributive lattice is discussed in [14] although originally in [17]. We refer to these works for notation and basic facts.

Proposition 7.5. For any commutative ring $\mathbf{A}$ there is a lattice $\mathbf{L}(\mathbf{A})$ such that $X=$ Spec $\mathbf{A}$ and Spec $\mathbf{L}(\mathbf{A})$ are canonically homeomorphic.

Proof. A basis for the topology of $X=\operatorname{Spec} A$ is the family of sets $X_{f}=\{x \in X: f \notin x\}$ where $f \in A$. These sets satisfy $X_{f} \cap X_{q}=X_{f g}$ for $f, g \in A$, and so finite unions of them, which we denote by $X_{u}=\bigcup\left\{X_{f}: f \in \mathbf{u}\right\}$ where $\mathbf{u}$ ranges over the set $\mathscr{F}(A)$ of finite subsets of $A$, form a distributive lattice. We denote this lattice by $\mathbf{L}(\mathbf{A})$ : it is the lattice of all quasi-compact open subsets of $X=\operatorname{Spec} A$. To prove the proposition we will show that the map

$$
x \rightarrow\left\{X_{u} \in L(A): x \notin X_{u}\right\}=P_{x}
$$

defines a homeomorphism from $X$ onto $\operatorname{Spec} \mathbf{L}(\mathbf{A})$. Clearly $P_{x}$ is a prime ideal of $\mathbf{L}(\mathbf{A})$. Conversely, if $P$ is a prime ideal of $\mathbf{L}(\mathbf{A})$ then $\left\{X_{u} \in L(A): X_{u} \notin P\right\}=\mathscr{E}$ is a family of quasi-compact open subsets of $X$; by a known result the easiest reference to which is [7], the family $\mathscr{E}$ has a unique point in its intersection. Call this $x$ and then $P=P_{x}$, as required. It remains to show that the exhibited bijection is in fact a homeomorphism. But this is easy since the sets $X_{f}=\{x \in X: f \notin x\}$, $(f \in A)$ and the sets $\left\{P_{x}: X_{f} \notin P_{x}\right\}$ correspond bijectively, each being a basis set for the respective topology. This concludes the proof.

Corollary 7.6. The spaces Minp A and Minp L(A) are canonically homeomorphic.

Proof. It is only necessary to remark that the subspace Minp A of Spec A can be identified by purely topological means (and similarly for $\mathbf{L}(\mathbf{A})$ ). But this is so because we can define the order $x \leqq y$ iff $y \in\{x\}^{-}$; MinpA is then the set of points of $X$ which are minimal in this order, equipped with the relative spectral topology. The order is preserved by the homeomorphism and so the corollary is proved. 
The corollary can be paraphrased as follows: if $\mathbf{A}$ is any commutative ring the space of minimal prime ideals of the lattice $\mathbf{L}(\mathbf{A})$ of quasi-compact open subsets of $X=\operatorname{Spec} \mathbf{A}$ is cannonically homeomorphic with Minp A. A precisely similar result is valid when $\mathbf{A}$ is replaced by any commutative $l$-group $\mathbf{G}$; although we do not give the (parallel) proof, we will use this fact in 7.12 below, and denote the lattice obtained by $\mathbf{L}(\mathbf{G})$.

\section{C. Illustrations, and the necessity of hypotheses}

EXAmple 7.7. Let $X$ be an infinite discrete space. Then $X$ is a zero-dimensional Baire space (since it is locally compact) which is not a $\zeta$-space; cf. 2.7, 2.8.

EXAMPLE 7.8. Let $X$ be the unit interval $[0,1]$ equipped with the usual topology. Then Minp $\mathbf{Z}(X)$ is an uncountably infinite discrete space by 7.4 ; in particular $\operatorname{Minp} \mathbf{Z}(X)$ is not compact, c.f. 6.1 above. Further, if $a \in \mathbf{Z}(X)$ is an infinite zero-set, then $\mathscr{M}_{\mathbf{Z}(\mathbf{x})}(a)$ is not compact cf. 5.5, 5.6 above.

EXAMPLE 7.9. Let $X=\beta N \backslash N$ where $N$ is the natural numbers equipped with the discrete topology. It is shown in [6] Example 5.9 that the ring $\mathbf{C}(\beta N \backslash N)$ is such that no point of Minp $\mathbf{C}(\beta N \backslash N)$ possesses a compact neighbourhood. Thus no point of the space $\operatorname{Minp} \mathbf{L}(\mathbf{C}(\beta N \backslash N)$ possesses a compact neighbourhood by 7.6; in particular Minp $\mathbf{L}(\mathbf{C}(\beta N \backslash N))$ is not locally compact, c.f. 5.8 above.

Example 7.10. Let $\mathbf{A}$ be the ring of Example 3.3 of [6]. There it is shown that Minp $\mathbf{A}$ is not countably compact, and so by $7.6 \operatorname{Minp} \mathbf{L}(\mathbf{A})$ is not countably compact, c.f. 5.10 above.

EXAMPLE 7.11. Let $\mathbf{G}$ be a conditionally $\sigma$-complete $l$-group without a weak order unit. Then $\operatorname{Minp} \mathbf{L}(\mathbf{G})$ is not compact although all of the sets $\mathscr{M}(x)$ are compact and the space is basically disconnected. Thus 6.7 represents a nontrivial extension of 6.3. Similarly, if $\mathbf{G}$ is a conditionally complete $l$-group without a weak order unit, Minp $\mathbf{L}(\mathbf{G})$ is not compact although all of the sets $\mathscr{M}(x)$ are compact, and the space is extremally disconnected. Thus 6.9 is a non-trivial extension of 6.6. These facts are proved in [16]. For $l$-groups with the stated properties, see [1].

To conclude the author would like to thank Mr. J. H. Rubinstein for permission to print the joint result 2.7, and the referee for his comments which led to the rewriting of a first attempt of this paper.

\section{References}

[1] G. Birkhoff, Lattice Theory (Amer. Math. Soc. Colloq. Publ. XXV 3rd Edn., Providence, Rhode Island, 1967).

[2] N. Bourbaki, Elements of Mathematics, General Topology Parts 1, 2 (Hermann, Paris, and Addison-Wesley, 1966). 
[3] N. Bourbaki, Eléments de Mathématique, Algebre Commutative Chapters 1, 2. (A. S. I. 1290, Hermann, Paris 1961).

[4] J. Dugundji, Topology (Allyn and Bacon Inc., Boston 1966).

[5] L. Gillman \& M. Jerison, Rings of Continuous Functions (D. Van Nostrand and Company Inc. Princeton N. J. 1960).

[6] M. Henriksen \& M. Jerison, 'The space of minimal prime ideals of a commutative rings', Trans. Amer. Math. Soc. 115 (1965), 110-130.

[7] M. Hochster, 'Prime Ideal Structure in Commutative Rings', Trans. Amer. Math. Soc. 142 (1969) 43-60.

[8] J. Kist, 'Minimal prime ideals in commutative semigroups', Proc. Lond. Math. Soc. (3) 13 (1963), 31-50.

[9] J. H. Rubinstein, 'Minimal prime ideals and compactifications', J. Austral. Math. Soc. 13 (1972) 423-432.

[10] R. Sikorski, Boolean Algebras. Second Edition (Springer-Verlag Berlin, 1964).

[11] T. P. Speed, 'A note on commutative semigroups', J. Austral. Math. Soc. 8 (1968), 731-6.

[12] T. P. Speed, 'Some remarks on a class of distributive lattices', J. Austral. Math. Soc. 9 (1969), 289-296.

[13] T. P. Speed, 'On Stone lattices', J. Austral. Math. Soc. 9 (1969), 297-307.

[14] T. P. Speed, 'Spaces of ideals of distributive lattices I, prime ideals', Bull. Soc. Roy. des Sci. de Liège $38^{\mathrm{C}}$ ann. (1969) 610-628.

[15] T. P. Speed, 'A note on commutative semigroups II', J. Lond. Math. Soc. (2) 2 (1970), 80-2.

[16] T. P. Speed, On lattice-ordered groups, Manuscript.

[17] M. H. Stone, 'Topological representation of distributive lattices and Brouwerian logics', Cas. pest. mat. fys. 1 (1937), 1-25.

[18] J. Varlet, 'On the characterisation of Stone lattices', Acta. Sci. Math. (Szeged) 27 (1966). $81-4$.

Department of Probability and Statistics

The University

Sheffield, S3 7RH

Engrand

Present address:

Department of Mathematics

University of Western Australia

W. A. 6009

Australia 